\title{
Análisis del turismo de salud en el área metropolitana de Bucaramanga con los países miembros de la Alianza del Pacifico ${ }^{1}$
}

DOI: http://dx.doi.org/10.17981/econcuc.37.2.2016.04

\section{Julio Ramírez Montañez ${ }^{2}$}

\begin{abstract}
Resumen
Para determinar las perspectivas y oportunidades del turismo de salud del Área Metropolitana de Bucaramanga, en el marco de los países miembros de Alianza del Pacifico como son México, Chile y Perú, se desarrolla el objetivo del presente artículo. A través de un análisis descriptivo se identifican las fortalezas de internacionalización de este sector, por lo que se considera los aspectos económicos y como referencia la medicina preventiva, la medicina curativa, la medicina estética y la medicina de bienestar.
\end{abstract}

Palabras clave: turismo de salud; Área Metropolitana de Bucaramanga; Alianza del Pacifico; industria médica de exportación.

Recibido: 9.9.2016 Devuelto para revisión: 7.11.2016 Aceptado: 29.11.2016

\begin{abstract}
${ }^{1}$ Artículo científico derivado de la investigación "Análisis del turismo de salud del Área Metropolitana de Bucaramanga con los Países Miembros de la Alianza del Pacifico", financiado por la Universidad Pontificia Bolivariana.

${ }^{2}$ Magister en Relaciones Internacionales, Flinders University of South Australia. Profesional en Relaciones Internacionales, Universidad Jorge Tadeo Lozano. Coordinador de Investigación, Escuela de Economía, Administración y Negocios. Investigador adscrito al grupo GRICANI de la Universidad Pontificia Bolivariana. Julio.ramirez@upb.edu.co
\end{abstract}




\title{
Analysis of health tourism in the metropolitan area of Bucaramanga with the member countries of the Pacific Alliance
}

\begin{abstract}
:
In order to determine the perspectives and opportunities of health tourism in the Metropolitan Area of Bucaramanga, the objective of this article is developed within the framework of the Pacific Alliance member countries such as Mexico, Chile and Peru. Through a descriptive analysis, the internationalization strengths of this sector are identified. Therefore, the economic aspects are considered and as a reference, preventive medicine, curative medicine, aesthetic medicine and welfare medicine.
\end{abstract}

Keywords: health Tourism; Metropolitan Area of Bucaramanga; Pacific Alliance; Medical Tourism Industry; Inspired Wellness.

\section{Introducción}

En la última década, Perú, Chile, México y Colombia han venido configurando una organización interestatal denominada Alianza del Pacífico, que se presenta como un nuevo esfuerzo de integración regional que, además de estimular in- 
tercambios comerciales y sinergias entre sus socios, pueda ser una herramienta para la inserción de empresas del sector turístico de salud del Área Metropolitana de Bucaramanga en la región.

En este mismo periodo de tiempo y como resultado del proceso de la globalización de mercados, la búsqueda de tratamientos y procedimientos médicos fuera de las fronteras nacionales por diferentes razones ha creado una oferta internacional de servicios médicos que combinan los procedimientos que buscan los pacientes con experiencias turísticas basadas en las riquezas naturales, culturales, artesanales y autóctonas de cada país oferente.

Ante la demanda de servicios de salud internacional, son varios los países que han institucionalizado una industria médica de exportación: Medical Tourism Industry, aprovechando sus ventajas competitivas para el fortalecimiento de esta actividad. Algunos de los más populares destinos de medicina turística que se destacan por la alta calidad a costos asequibles con referencia a Estados Unidos y países desarrollados son: Brasil, Costa Rica, Cuba, India, Malasia, México, Panamá, Filipinas, Sudáfrica, Tailandia y Turquía (MinTIC, 2010).
La referencia anterior permite apreciar cómo el mercado del turismo médico ha crecido de manera importante en cada uno de los países que lo han involucrado como una de sus políticas de competitividad nacional. La firma consultora McKinsey \& Company determinó que el sector de turismo de salud en Colombia es aún incipiente con una oferta de valor concentrada en la medicina curativa y estética con un flujo de entre 4.100 y 7.000 pacientes de las categorías de medicina y 44.800 en busca de procedimientos de bienestar (inspired wellness). Según este informe, para capturar valor en este sector, el país requiere cerrar brechas, principalmente en percepción de calidad, cantidad de personal trabajando en el sector, bilingüismo e infraestructura (McKinsey \& Company, 2010).

En este sentido, empresarios visionarios del sector salud del Área Metropolitana de Bucaramanga han venido trabajando arduamente en el objetivo de posicionarse competitivamente con miras a la exportación de servicios médicos y odontológicos, caracterizados por los procesos de investigación y desarrollo en procedimientos médicos especializados que pudieran tener oportunidades en marco del acuerdo comercial de la Alianza del Pacífico. 


\section{Fundamento teórico}

El turismo de salud recibe varias denominaciones en el ámbito internacional, como turismo médico, medical tourism, turismo de bisturí, viajes médicos, viajes de salud, viajes para tratamiento de salud, pero la más conocida y aceptada internacionalmente es turismo de salud. En el mundo los principales países que manejan el tema son Tailandia, Singapur, India, Costa Rica, Sudáfrica, México, Brasil y Estados Unidos.

Para definir el término turismo de salud se debe señalar inicialmente la enunciación de turismo emitida por la Organización Mundial del Turismo (OMT), la cual tras varios años de discusiones respecto al verdadero sentido de esta palabra y los aspectos que la componen, concluyó:

El turismo comprende las actividades que realizan las personas durante sus viajes y estancias en lugares distintos al de su entorno habitual, por un periodo de tiempo consecutivo inferior a un año con fines de ocio, por negocios y otros (OMT, 1994).

Por su parte, la Organización Mundial de la Salud (1946), señala que el concepto de salud:
Está relacionado con un estado de completo bienestar físico, mental y social; y no solo la ausencia de afecciones y enfermedades (OMS, 1946).

Una vez presentadas las definiciones anteriores de turismo y salud emitidas por la Organización Mundial del Turismo y la Organización Mundial de la Salud, se realizará a continuación una aproximación conceptual sobre el turismo de salud y sus diversas vertientes.

Existen diversas definiciones del concepto de turismo de salud. La Organización Mundial del Turismo define turismo de salud como "el proceso en el cual una persona viaja para recibir servicios de salud en un país diferente al país que reside" (OMT, 2012).

Por su parte, el Ministerio de Comercio, Industria y Turismo de Colombia define el turismo de salud, "como la exportación de servicios de salud enfocado en 4 áreas específicas: medicina curativa, preventiva, estética y de bienestar" (MinCIT, 2009).

De igual forma, Keckley y Underwood (2008) definen el turismo de salud "como el acto de desplazarse desde el sitio de residencia de una persona, hacia el exterior o hacia otra ciudad de su país, para recibir servicios de salud o de bienestar". 


\section{Metodología}

El presente estudio tiene aspectos explicativos y descriptivos; exploratorios por cuanto se requiere obtener datos sobre la dinámica comercial del sector turismo de Colombia, México, Chile y Perú. Es de igual forma descriptivo porque integra el conjunto de indicadores para determinar las oportunidades del sector de turismo de salud en los países miembros de la Alianza del Pacífico.

Fase 1. Caracterización del sector turismo de salud en los países de la Alianza del Pacífico. Incluye la revisión bibliográfica y apropiación de conceptos y aspectos técnicos para la obtención y el manejo de cifras.

Fase 2. Reconocimiento y evaluación de las diferentes fuentes que generan datos sobre la determinación de la estructura productiva del sector de turismo de salud en Colombia, México, Chile y Perú para seleccionar las fuentes más apropiadas para la toma de información.

Fase 3. Análisis de la legislación y las políticas públicas del sector de turismo de salud de los países miembros de la Alianza del Pacífico.
Fase 4. Análisis de la información: análisis de cada conjunto de indicadores y de la integración de los mismos para los productos del sector. Procurar una conclusión general del posicionamiento competitivo del sector de turismo de salud.

\section{Resultados}

Un reciente estudio realizado por McKinsey \& Company (2009) señala que los servicios de turismo de salud se dividen en 4 categorías:

Medicina curativa. Ésta promueve la buena salud de las personas a través de múltiples modalidades a tratar, entender y mejorar la calidad de vida con una enfermedad. Busca eliminar una enfermedad o condición médica desfavorable preexistente y tratar una condición médica específica, promoviendo la buena salud de las personas. Los tratamientos y procedimientos más sobresalientes son el manejo de artritis, dolores crónicos, trasplantes, cirugía vascular, entre otros.

Medicina preventiva. Busca prevenir futuras apariciones de enfermedades optimizando la salud a través de transiciones. 
Los tratamientos más sobresalientes son los chequeos ejecutivos, tratamientos de traumatologías ortopédicas de baja complejidad, entre otros.

Medicina estética. Busca la satisfacción de la apariencia física. Los tratamientos más sobresalientes son la cirugía plásticas con fines estéticos como mamoplastia, rinoplastia y liposucción, tratamientos odontológicos, acné y soluciones a desordenes de pigmento, tratamiento de belleza alternativo, bótox.

Bienestar (Inspired Wellness). Busca la satisfacción física, espiritual y mental de los pacientes. Este campo se ha desarrollado a partir de la necesidad del manejo de estrés y sobrepeso. Los tratamientos más sobresalientes son la exploración por medio de la meditación, talleres de nutrición, programas de bienestar para toda la familia y talleres de estilo de vida (McKinsey \& Company, 2010).

Esta caracterización de los servicios de turismo de salud se presenta de manera más detallada en la figura 1.

Sobre los principales modos de prestación de servicios, se estableció que la prestación internacional de servicios de salud se realiza a través de cuatro modos definidos en el Acuerdo General sobre el Comercio de Servicios (AGCS):

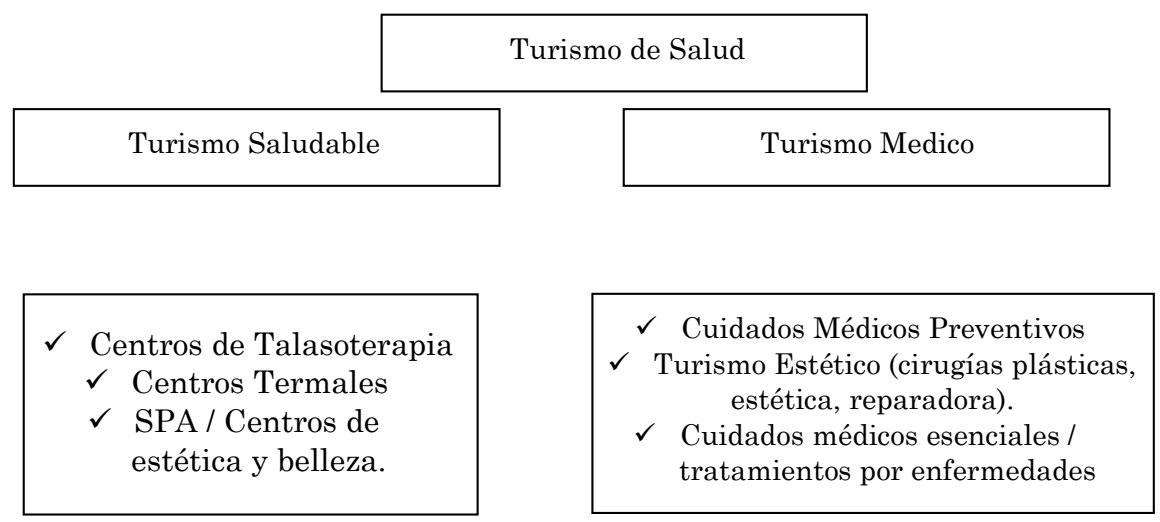

Fig. 1. Turismo de salud.

Fuente: Elaboración propia a partir de McKinsey \& Company, 2010. 
- Modo 1. Prestación transfronteriza de servicios.

- Modo 2. Movimiento de pacientes. Ha permitido a pacientes de países desarrollados recurrir a los servicios y tratamientos ofrecidos por países en desarrollo a precios competitivos. El principal obstáculo es la no inclusión de estos servicios en las pólizas de seguros.

- Modo 3. Presencia comercial de prestadores extranjeros. Restricciones de los países a limitar el ingreso de suministradores extranjeros.

- Modo 4. Migración temporal de profesionales. Cupos esporádicos por cantidades determinadas de profesionales o enfermeras, y por tiempos limitados dependiendo de la necesidad de los países; limitación en la consecución de visas (DNP, 2007).

De igual forma, es importante explicar la forma en la cual se desarrolla la industria del turismo de salud en el mundo, pues este tipo de negocio se desarrolla de forma particular. La figura del turismo de salud ha cambiado los parámetros tradicionales asociados a la prestación trasnacional de estos servicios. En otras épocas, los habitantes de países con bajos y medianos ingresos se trasladaban a los países industrializados en busca de atención especializada. En la actualidad, muchos de los países del primer grupo se han hecho famosos por su buena atención médica, tratamiento de enfermedades y rehabilitación, cirugía y otros procesos ofrecidos a costos mucho más reducidos que aquellos que ofrecen los países de origen de los turistas.

Los países en vías de desarrollo han visto en el turismo de la salud una fuente de importantes ingresos y lo están promoviendo agresivamente, por tanto, vienen impulsando la competencia en esta industria. Sin embargo, es importante garantizar la protección de los turistas de la salud con la elaboración de las directrices apropiadas y los procedimientos de certificación correspondientes (Arias, Caraballo y Matos, 2012).

En los países donde se implementa el turismo de salud, se tienen como compradores a los clientes individuales, por un lado, que son los pacientes que necesitan y buscan algún tipo de tratamiento médico y, por otro, a los clientes corporativos, compuestos por empresas que refieren pacientes al proveedor médico y se clasifican en tres grandes grupos: compañías aseguradoras, compañías auto-aseguradoras $\mathrm{y}$ facilitadores médicos. 
Las compañías aseguradoras son partícipes de foros regionales y de visitas a clínicas y hospitales para la identificación y selección de proveedores médicos y comprender la dinámica del envío de pacientes al exterior. Por su parte, las compañías auto-aseguradas constituidas por empresas, organizaciones y corporaciones tanto públicas como privadas que poseen sus propios planes de beneficios para sus empleados y familiares, se interesan en el turismo médico por las mejores condiciones de cobertura, beneficios, reducción de costos y ampliación de los programas a familiares. Finalmente, se encuentran los facilitadores médicos, que son los principales promotores y comercializadores a nivel mundial para atraer turistas de salud y lo conforman empresas que poseen su propia red de clínicas y hospitales en diferentes países.

En cuanto a las últimas tendencias del turismo de salud, tomando como referencia destinos, tratamientos y oferta de servicios, McKinsey \& Company (2012) presenta el siguiente registro:

\section{Principales destinos de los turistas} de salud en el mundo. Asia y Norteamérica atraen el mayor flujo de turistas de salud en busca de servicios en las categorías de medicina. En el caso de la categoría de bienestar (inspired wellness), Medio Orien- te y América Latina son los destinos más atractivos para los turistas internacionales.

Tratamientos más buscados en el mundo. Según McKinsey \& Company, para el 2015 se estima que los tratamientos que ofrecerán mayor rentabilidad y atractivo estarán asociados con la categoría de medicina preventiva y de bienestar (p.ej. manejo de peso, salud masculina $\mathrm{y}$ manejo de estrés).

Oferta de servicios en el ámbito mundial. En la categoría de medicina, en América Latina la especialidad con mayor oferta es la de cirugía estética, en Europa es la rehabilitación, en Asia la cardiología y en Medio Oriente la oncología. En bienestar, la oferta de spas está concentrada en Europa, Norte América y Asia, tanto en número de spas como en ingresos (McKinsey \& Company, 2010).

De igual forma, la acreditación internacional en salud es un elemento que en la actualidad ha tomado mayor relevancia, ya que representa reconocimiento internacional para los proveedores de servicios médicos porque conlleva a la buena percepción de los compradores y, por ende, a una buena imagen.

En el contexto regional, el Instituto Colombiano de Normas Técnicas y Certifica- 
ción ICONTEC, como entidad acreditadora, promueve la participación voluntaria de las organizaciones de salud para que realicen sus procesos de autoevaluación y evaluación para una mejor gestión y desempeño que propenda por una mayor productividad y óptima utilización de los recursos y así beneficiar a los afiliados, beneficiarios y usuarios de los servicios de salud (ICONTEC, 2012).

La Joint Commission International (JCI) es líder mundial en la acreditación de organizaciones de atención de la salud y es una de las empresas acreditadoras más conocidas en América Latina. En 2005, la Organización Mundial de la Salud designó a la JCI como su único Centro Colaborador para Soluciones en Seguridad del Paciente para promover una atención segura y de alta calidad. Contar con la acreditación de la JCI significa tener compromiso con la excelencia en la seguridad y la atención del paciente, lo que le permite reconocer que su servicios están a la altura de las prácticas internacionales más importantes (JCI, 2012).

Estas instituciones verifican el cumplimiento de unas condiciones claves que debe tener un destino turístico de salud de clase mundial. Cárdenas y Linares (2010a) plantean que en el ámbito internacional existen unas condiciones claves para la atención de pacientes. El cumplimiento de estas condiciones por parte de las instituciones prestadoras de salud interesadas en lograr su inclusión en redes internacionales para la atención de pacientes, les da ventajas competitivas y las hace ser atractivas para acceder a ese mercado.

Cárdenas y Linares (2010b) hacen un listado de las condiciones mínimas con las cuales debe contar un destino turístico de salud para ser considerado de talla mundial. Estas condiciones son:

- Las instituciones prestadoras de salud deben contar con acreditación internacional. Cumplimiento de estándares de calidad y seguridad del paciente; igualmente, infraestructura moderna y tecnología de punta.

- Manejo impecable del idioma inglés. Esto para el personal asistencial y administrativo que intervenga en el proceso de atención del paciente, historia clínica, señalización hospitalaria, página web, material de educación para los pacientes, contratos, programación de televisión, entre otros.

- Contar con una oficina para atención de pacientes internacionales. Para garantizar el acceso directo y con oportunidad del servicio de salud programado. 
- Contar con certificación de los profesionales de la salud. Estos profesionales deben cumplir con la idoneidad, las competencias y el entrenamiento requerido.

- Tener centros de excelencia. Tener un programa implementado de salud cuya oferta de valor se sustente en resultados clínicos y niveles de seguridad del paciente comparables con los mejores referentes internacionales a unos costos muy competitivos en el mercado para un procedimiento o condición de salud específica, utilizando la mejor evidencia científica disponible.

- Humanismo. El personal que interviene en la atención de los pacientes debe tener disposición para el servicio, amabilidad, compromiso y sensibilidad cultural y religiosa.

- Historia clínica en inglés. Cuando el paciente es dado de alta se debe hacer entrega de la historia clínica traducida al inglés y hacerse un envío al médico tratante o institución responsable para los cuidados posteriores; asimismo, debe haber disposición para su consulta a cualquier hora y a través de diferentes tecnologías.
- Contar con tecnologías en salud (eHealth). Se debe proveer un servicio de telemedicina para ver y acompañar intervenciones médicas en tiempo real; video de la intervención quirúrgica (médica u odontológica).

Turismo de salud en Colombia. En el ámbito nacional, según el informe presentado por el Ministerio de Comercio Industria y Turismo de Colombia (MinCIT) titulado Desarrollando sectores de clase mundial, el sector de turismo de salud está compuesto por 4 categorías básicas (medicina curativa, preventiva, estética y bienestar) en las que Colombia podría competir con una propuesta estratégica clara, capturando al menos 2,8 millones de turistas de salud y generando ingresos por al menos 6,3 mil millones de dólares en el año 2032 (MinCIT, 2009).

El mejoramiento de la infraestructura ha sido un punto a favor del sector de turismo de salud de Colombia. Según el Departamento Administrativo Nacional de Estadística (DANE, 2014), el total de área de construcción de centros de salud (hospitales de alta complejidad) en las siete principales ciudades del país (Bogotá, Medellín, Cali, Barranquilla, Bucaramanga, Pereira y Armenia) creció de forma sostenida desde el 2005. 
En el último trimestre del 2005 había $119.000 \mathrm{~m} 2$ en construcción, mientras que para el segundo trimestre del 2014 existían $707.000 \mathrm{~m} 2$ en construcción, logrando así un crecimiento de $494 \%$.

Por su parte, el informe Health and Wellness Tourism in Colombia destaca que "Colombia es uno de los más atractivos destinos para el turismo de salud y bienestar en Latinoamérica por las altas calificaciones de sus profesionales en el área de salud y el bajo costo de los procedimientos con respecto al de sus países de origen" (Euromonitor, 2014). Esta situación generó un incremento en el flujo de visitantes extranjeros en más de 50.000 en el año 2013, siendo los procedimientos más demandados los de cirugía de corazón, cirugía bariátrica y cirugías de ojos.

En ese mismo sentido, la publicación de Proexport titulada La Revista de las Oportunidades Proexport Colombia, Santander Aprovecha los TLC señala que "en la actualidad, los consumidores extranjeros de servicios médicos nacionales son en su mayoría colombianos residentes en Estados Unidos. En segunda instancia, latinos o hispanoparlantes que tienen relación con Colombia" (Proexport, 2012). Este estudio, determina que Colombia compite con sus países vecinos Costa Rica, Panamá, y México, entre otros, en la gran mayoría de los procedimientos médicos. A la hora de tomar la decisión, los consumidores tienen en cuenta las medidas relacionadas con la seguridad del paciente, tasas de infección y acreditaciones internacionales de los hospitales.

La oferta de servicios que Colombia ofrece está enfocada en la reproducción, oncología, oftalmología, cirugía plástica y reconstructiva, estudios y procedimientos con células madres, cardiología, cirugía bariátrica (obesidad), tratamientos dentales y chequeos médicos ejecutivos, entre otras especializaciones.

Para procedimientos de baja complejidad (tratamientos dentales, oftalmológicos o cirugías plásticas), la decisión se toma por recomendación o publicidad. En el caso de operaciones complejas y costosas hay empresas dedicadas a intermediar entre los consumidores y los proveedores de salud, que prestan un servicio integral desde la facilitación de consultas preoperatorias hasta la logística de viaje para el paciente y su familia (Proexport, 2012).

En la tabla 1 se presenta la dinámica de ventas del sector de turismo de salud en Colombia en el periodo 2008-2013, donde se pone en evidencia una evolución progresiva en todos los indicadores analizados. 
Tabla 1

Ventas del sector de turismo de salud y bienestar de Colombia en el periodo 2008-2013.

\begin{tabular}{ccccccc}
\hline${ }^{*}$ Miles de millones de pesos & 2008 & 2009 & 2010 & 2011 & 2012 & 2013 \\
\hline Turismo médico & 120,5 & 139,7 & 164,4 & 181,2 & 203,1 & 229,1 \\
Spas & 139,8 & 158,6 & 181,3 & 197,7 & 212,6 & 229,2 \\
Spas & 24,3 & 27,6 & 31,6 & 34,6 & 37,4 & 40,5 \\
Hotel Resort Spa & 97,9 & 111,1 & 126,9 & 138,4 & 148,9 & 160,5 \\
Otros Spas & 17,6 & 19,9 & 22,8 & 24,7 & 26,3 & 28,2 \\
Turismo de salud y bienestar & 260,3 & 298,3 & 345,7 & 378,9 & 415,7 & 458,3 \\
\hline
\end{tabular}

Fuente: Euromonitor, 2014.

En la tabla 1 se puede apreciar la progresiva evolución de las ventas totales del sector, pasando de vender $\$ 260.000$ millones en el año 2008 a vender $\$ 458.300 \mathrm{mi}-$ llones, presentándose un incremento de un $56 \%$ en el periodo de estudio. De igual forma, es de destacar la evolución en casi 52\% de las ventas de turismo médico pasando de $\$ 120.000$ millones a $\$ 229.000$ millones en el mismo periodo.

\section{Perspectivas del sector de turismo} de salud en Colombia. Los países que invierten en el sector del turismo de salud en Colombia son Estados Unidos con un $42 \%$, España con un $18 \%$, Chile con un $12 \%$, Brasil con un $13 \%$, la inversión por parte de nacionales en el sector ronda el
$13 \%$, mientras que el restante $2 \%$ pertenece a otros orígenes.

Las inversiones en el sector de turismo de salud son progresivas en el año 2013, la inversión en infraestructura clínica, hospitalaria y estética aumentó un 120\% respecto a 2012 (Proexport, 2013). Las inversiones se han caracterizado por buscar brindar un servicio de alta calidad, principalmente dirigido a pacientes internacionales que buscan realizarse tratamiento e intervenciones (invasivas o no invasivas), ya sea para fines curativos, preventivos, estéticos o de bienestar integral.

De acuerdo con el informe Health and Wellness Tourism in Colombia, en el año 
2013 el turismo de salud y bienestar en Colombia creció en un $10 \%$ con respecto al año anterior con un monto de $\$ 458.000$ millones de pesos colombianos. El turismo médico continúa teniendo la mayor participación en este monto.

De igual forma, las perspectivas según el sector a nivel nacional son las mejores. De acuerdo con previsiones realizadas por la Asociación Colombiana de Turismo de Salud (2012), para el año 2020, el sector de turismo de salud manejaría las siguientes cifras (Tabla 2):
Turismo de salud en los países de la Alianza del Pacífico. A continuación se presenta una breve descripción del sector de turismo de salud en México, Perú y Chile.

Turismo de salud en México. De acuerdo con el estudio titulado Health and Wellness Tourism in Mexico, este país se ha posicionado como uno de los destinos preferidos de turismo de salud en el mundo. Su cercanía a Estados Unidos y sus costos en un 50\% más bajo en cirugías y procedimientos médicos lo hacen atractivo especialmente para los Estadounidenses (Euromonitor, 2014).

Tabla 2

Previsión de crecimiento de turistas de salud de Colombia en el mercado mundial para 2020.

\begin{tabular}{|c|c|c|c|c|c|}
\hline Categoría & $\begin{array}{l}\text { Turistas } \\
\text { de salud en } \\
2014 \text { a nivel } \\
\text { mundial }\end{array}$ & $\begin{array}{c}\text { Turistas } \\
\text { de salud } \\
\text { en } 2014 \text { en } \\
\text { Colombia }\end{array}$ & $\begin{array}{c}\text { Previsión de } \\
\text { participación global } \\
\text { para Colombia: } \\
2015-2020\end{array}$ & $\begin{array}{l}\text { Crecimiento } \\
\text { interanual }\end{array}$ & $\begin{array}{c}\text { Previsión de } \\
\text { turistas de } \\
\text { salud para } \\
2020\end{array}$ \\
\hline $\begin{array}{c}\text { Turistas de } \\
\text { medicina } \\
\text { curativa }\end{array}$ & 710.000 & 5.400 & $5,00 \%$ & $14,36 \%$ & 355.00 \\
\hline $\begin{array}{c}\text { Turistas de } \\
\text { medicina } \\
\text { estética y } \\
\text { preventiva }\end{array}$ & 2.600 .000 & 4.300 & $5,00 \%$ & $16,11 \%$ & 130.000 \\
\hline $\begin{array}{c}\text { Turistas } \\
\text { de salud de } \\
\text { bienestar } \\
\text { (inspired } \\
\text { wellness) }\end{array}$ & 7.210 .0000 & 52.000 & $4,00 \%$ & $16,36 \%$ & 2.884 .000 \\
\hline
\end{tabular}

Fuente: Asociación Colombiana de Turismo de Salud, Informe de McKinsey Mapping The Global Tourism Market, 2012. 
México cuenta con más de 100 hospitales certificados con estándares internacionales en áreas de la medicina alternativa como hidroterapia, temazcal y talasoterapia, entre otras. Esto ha sido una iniciativa conjunta del sector público y privado invirtiendo en los últimos años en hospitales y hoteles en las ciudades de Monterrey, Puebla, IxtapaZihuatanejo, Puerto Vallarta, Querétaro, San Luis Colorado, Riviera Maya, Tijuana y Ciudad de México. Su propósito es ampliar y mejorar la infraestructura y ofrecer servicios de alta calidad, en orden de atraer más pacientes e incrementar el tiempo de permanencia en el país. Algunos de estos proyectos son los conocidos como Ciudad de la salud donde se incluyen todos los servicios que los pacientes y sus familiares puedan necesitar (hospedaje, transporte y atracciones, entre otras).

En México son numerosos los estados que practican y promocionan el turismo de salud por muchas circunstancias favorables, como por ejemplo la vecindad con los Estados Unidos; sin embargo, en América Latina sobresale como uno de los tres principales destinos de salud sólo a través de Monterrey, Ciudad de la salud.

El informe de Euromonitor también señala que en el año 2013 las ventas del sector de salud y servicios de México se incrementaron en un 9\% con respecto al año anterior llegando a los 53 billones de pesos mexicanos y el número de resort spas aumentó en 469 (Euromonitor, 2014).

Tabla 3

Ventas del sector de turismo de salud y bienestar de México en el periodo 2008-2013.

\begin{tabular}{cccccccc}
\hline * Millones de pesos mexicanos $M X N$ & 2008 & 2009 & 2010 & 2011 & 2012 & 2013 \\
\hline Turismo médico & 19.869 & 19.630 & 20.796 & 26.581 & 34.654 & 37.740 \\
Spas & 12.271 & 12.653 & 12.507 & 12.416 & 14.027 & 15.215 \\
Spas & 2.860 & 2.959 & 2.909 & 3.229 & 3.487 & 3.805 \\
Hotel Resort Spa & 8.290 & 8.451 & 8.451 & 8.992 & 9.316 & 10.088 \\
Otros Spas & 1.119 & 1.146 & 1.146 & 1.194 & 1.233 & 1.322 \\
Turismo de salud y bienestar & 32.140 & 33.304 & 33.304 & 39.997 & 48.681 & 52.956 \\
\hline
\end{tabular}

Fuente: Euromonitor, 2014. 
En la actualidad, algunos de los principales procedimientos que se adelantan en México son cirugía plástica, tratamientos de oncología, fertilidad, oftalmología, cardiológicos y odontológicos. Otro tipo de tratamientos preferidos por los Estadunidenses en México son los de turismo de bienestar (wellness) por los cientos de spas y hoteles spa que ofrecen este tipo de servicios.

En la tabla 3 se presenta la evolución en las ventas del sector de turismo de salud en el periodo 2008-2013. Poniendo en evidencia la tendencia positiva en las ventas del sector de turismo de salud de México en el periodo 2008-2013. Esta tendencia tiene un incremento porcentual del $60 \%$ pasando de MXN $\$ 32.140$ en el año 2008 a vender MXN $\$ 52.956$.
El turismo médico también tiene un alto crecimiento porcentual, llegando al 54\% en el periodo al pasar de vender MXN $\$ 19.869$ en el año 2008 a vender MXN $\$ 37.740$.

Turismo de salud en Perú. De acuerdo con el informe titulado Health and Wellness Tourism in Perú de agosto del 2014, el turismo médico se incrementó en Perú en un 14\% en el año 2013, después del aumento del $5 \%$ registrado en el año 2012 . La mayoría de turistas viajan al Perú en búsqueda de tratamientos dentales, cirugías plásticas, operaciones con láser y tratamientos de fertilidad. La mayoría de pacientes provienen de Chile, España y Estados Unidos.

Tabla 4

Ventas del sector de turismo de salud y bienestar de Perú en el periodo 2008-2013.

\begin{tabular}{cccccccc}
\hline * Millones de nuevos soles PEN & 2008 & 2009 & 2010 & 2011 & 2012 & 2013 \\
\hline Turismo médico & 443.7 & 469 & 512.1 & 559.3 & 571.9 & 654 \\
Spas & 103.5 & 109 & 136.6 & 149.1 & 171.6 & 194.2 \\
Destination Spas & 13.5 & 16.4 & 24.6 & 26.7 & 29.2 & 31.1 & 90.9 \\
Hotel Resort Spa & 60 & 62.4 & 75.1 & 80.5 & 97.1 & 51.5 & 66 \\
Otros Spas & 30 & 30.6 & 36.9 & 41.9 & 743 & 848 \\
\hline
\end{tabular}

Fuente: Euromonitor, 2014. 
Según Euromonitor (2014), tres aspectos hacen de Perú un destino médico ideal. Primero, la infraestructura médica privada ha mejorado sustancialmente en los últimos años con la construcción de nuevas clínicas con equipos de última tecnología; segundo, la excelencia en los servicios médicos y la reputación ganada en los últimos años en los tratamientos médicos; y tercero, sus costos son bajos en comparación con otros países.

Los más importantes proyectos en los próximos años están enfocados a mejorar las condiciones de oferta de servicios de ecoturismo, turismo espiritual y turismo social. En ese sentido, sin embargo, Perú busca proyectarse internacionalmente como un sector sostenible y responsable con el medio ambiente, además de posicionarse como un país líder a nivel Latinoamericano en materia de termalismo; por lo tanto, podría considerarse como un nuevo competidor en el sector de turismo de salud y, específicamente, en la categoría de bienestar o turismo de bienestar.

Turismo de salud en Chile. De acuerdo con el informe titulado Health and Wellness Tourism in Chile de agosto del 2014, el turismo de salud en Chile creció en el año 2013, especialmente por la alta oferta de descuentos que se encuentran en internet, donde se ofrece transporte, hospedaje y servicios de spa como parte del paquete turístico con los tratamientos médicos ofrecidos en las clínicas Alemana y Las Condes, ubicadas en Santiago (Euromonitor, 2014).

Tabla 5

Ventas del sector de turismo de salud y bienestar de Chile en el periodo 2008-2013.

\begin{tabular}{ccccccc}
\hline * Millones de pesos chilenos CLP & 2008 & 2009 & 2010 & 2011 & 2012 & 2013 \\
\hline Turismo médico & 25.345 & 26.000 & 27.845 & 29.419 & 31.415 & 33.412 \\
Spas & 59.602 & 55.285 & 62.171 & 64.792 & 65.792 & 68.423 \\
Destination Spas & $-\ldots$ & $-\ldots-$. & 4.443 & 4.298 & 4.928 & 5.399 \\
Hotel Resort Spa & 30.406 & 31.522 & 32.981 & 34.283 & 34.755 & 36.423 \\
Otros spas & 29.195 & 23.762 & 24.747 & 26.108 & 26.108 & 26.600 \\
Turismo de salud y bienestar & 84.947 & 81.266 & 90.016 & 97.207 & 97.207 & 101.836 \\
\hline
\end{tabular}

Fuente: Euromonitor, 2014. 
La Clínica Los Condes recibió más de 6.000 hospitalizaciones de pacientes extranjeros en el año 2013. De esos, 1.000 viajaron desde sus países de orígen especialmente desde Bolivia y Estados Unidos. La Clínica Los Condes vendió paquetes de medicina preventiva en Bolivia, Perú, Paraguay, Uruguay y Ecuador, los cuales incluyen pasajes y hospedaje en Santiago. La Clínica Alemana por su parte, recibe más de 8.000 pacientes al año, quienes principalmente arriban desde Bolivia, Paraguay, el Reino Unido, Estados Unidos y España buscando tratamientos de cirugía plástica, reducción de peso, tratamientos de cáncer, chequeos preventivos, problemas neurológicos, accidentes cardiovasculares, urología y traumatología (Euromonitor, 2014).

Termas Puyehue Wellness \& Spa Resort, que opera desde 2010, es el principal prestador de servicios turísticos de salud. Este spa, ubicado en la décima región, cuenta con alrededor de 107.000 hectáreas de bosque denominada reserva de biosfera por la UNESCO. La compañía ofrece servicios de hospedaje, alimentación, transporte, actividades al aire libre, sauna, terapias termales y deportes.

Según Euromonitor, el turismo médico representa una gran oportunidad para
Chile con excelentes clínicas, médicos y precios competitivos en términos internacionales. Se dice que Chile es nuevo en la industria de turismo de salud pero que tiene gran potencial en países con menor desarrollo en la medicina como Bolivia y Perú (Euromonitor, 2014).

\section{Conclusiones}

Dentro del proceso de internacionalización del turismo de salud del Área Metropolitana de Bucaramanga se destacan varios proyectos desarrollados en la región, como el tratamiento para niños con enfermedades congénitas de corazón. Estos pacientes arriban principalmente desde Ecuador, Venezuela, Estados Unidos y Canadá. Otro proyecto importante es el ofrecido por la Fundación Cardiovascular (FCV) el cual es un programa líder a nivel mundial, llamado Centro Nacional de Telemedicina que consta de una red de más de cien hospitales vinculados que están conectados por medio de un telecentro las 24 horas al día para tratar pacientes de poblaciones apartadas

Pero tal vez el megaproyecto más ambicioso es el conocido como Zona Franca Permanente Especial FOSUNAB, emprendido conjuntamente entre la Fundación Oftalmológica de Santander y la Univer- 
sidad Autónoma de Bucaramanga, donde se integraran servicios médicos, hospitalarios, de innovación, de investigación científica y desarrollo de conocimiento mediante la implementación de unidades de servicio de alta especialización en las áreas de cirugía estética, tratamiento de cáncer, cardiovascular y banco de células madre, además, tendrá servicios de un hospital cinco estrellas, un centro de convenciones y un helipuerto.

Son múltiples los retos para el sector de turismo de salud del Área Metropolitana de Bucaramanga frente a sus competidores de México, Chile y Perú en el marco de los acuerdos celebrados con estos países en el contexto de la Alianza del Pacífico. Es claro que para algunas empresas de salud, la ampliación de los mercados y el afianzamiento de los vínculos con estos países pueden ser una oportunidad para alcanzar importantes metas, como la ampliación en los volúmenes de sus ventas y una mayor estabilidad financiera.

No obstante, también se presenta una amenaza para algunos servicios de salud del Área Metropolitana de Bucaramanga debido a que México, Chile y Perú pueden ofrecer servicios con mayor reconocimiento por parte de los turistas, pudiendo afectar a la industria regional de este sector. De igual forma, persisten limitaciones que pudieran amenazar la proyección del turismo de salud del Área Metropolitana de Bucaramanga; algunos aspectos importantes son: el bajo porcentaje de profesionales de salud que domina el idioma inglés, la percepción de inseguridad en el país que aún persiste a pesar de encontrarse en una negociación para llegar a un acuerdo de paz y la falta de un reconocimiento mundial del turismo del sector en Colombia. Los anteriores representan un desafío que va más allá de la planeación gubernamental para transformarse en una visión estratégica de desarrollo económico regional que apoye al sector privado en el largo plazo.

En ese sentido, esta investigación pretende determinar las perspectivas y oportunidades que pudiera ofrecer para el sector de turismo de salud del Área Metropolitana de Bucaramanga en el marco del acuerdo comercial de la Alianza del Pacífico con los países de México, Chile y Perú.

\section{Referencias}

Arias, F., Caraballo, A. y Matos, R. (2012). El turismo de salud: Conceptualización, historia, desarrollo y estado actual del mercado global. Clío América, 6(11). 72-98. 
Asociación Colombiana de Turismo de Salud - ACTUS. (2012). Mapping the Global Tourism Market. Informe McKinsey.

Cárdenas, C. y Linares, N. (2010a). Informe de visita de la CEO de Mercury Healthcare Maria K Todd, a las Instituciones Prestadoras de Servicios de Salud del Cluster Servicios de Medicina y Odontología de Medellín. Visita del facilitador de Turismo Médico a tres IPS del Cluster Servicios de Medicina y Odontología de Medellín. Único: 1-8.

Cárdenas, C. y Linares, N. (2010b). Registro de atención de pacientes internacionales en el período 1 de junio al 31 de diciembre de 2009, realizado con los reportes de las Instituciones Prestadoras de Servicios de Salud - IPS del Cluster Servicios de Medicina y Odontología de Medellín. Registro de atención de pacientes internacionales en 2009. Cluster Servicios de Medicina y Odontología de Medellín. Único.

Euromonitor. (2014). World Retail Data and Statistics 2014. Recuperado de http://www.euromonitor.com/medialibrary/PDF/Book_WRDAS_2014. pdf
Instituto Colombiano de Normas Técnicas y Certificación ICONTEC. (2012). Marco filosófico de ICONTEC como entidad acreditadora. Recuperado de: http:// www.acreditacionensalud.org.co/acreditacion.php?IdSub=116\&IdCat $=29$

Joint Commission International - JCI. (2012). Centro Colaborador de la OMS para Soluciones en la Seguridad del Paciente. Recuperado de: http:// es.jointcommissioninternational.org/ enes/WHO-Collaborating-Centre-forPatient-Safety-Solutions/

Keckley, P. y Underwood, H. (2008). Medical Tourism: Consumers in Search of Value. Washington: Deloitte Center for Health Solutions.

McKinsey \& Company. (2009). Desarrollando sectores de clase mundial en Colombia Informe final sector turismo de salud. Bogotá: McKinsey Pub.

McKinsey \& Company. (2010). Informe de Modelo de demanda de turismo de salud para 2015. Buenos Aires: McKinsey.

McKinsey \& Company. (2012). Mapping the Market for Medical Travel. Boston: McKinsey.

McKinsey \& Company. (2013). Informe Sobre Turismo de Salud en India para 2014. Bombay: McKinsey. 
Organización de las Naciones Unidas. OMS - Organización Mundial del Trabajo. (1946). Constitución de la Organización Mundial de la Salud. Recuperado de http://www.who.int/governance/eb/ who_constitution_sp.pdf?ua=1

Organización de las Naciones Unidas. OMT - Organización Mundial del Trabajo. (1995). Introducción al Turismo. Recuperado de http://www.e-unwto.org/doi/ pdf/10.18111/9789284402694

Organización de las Naciones Unidas. OMT - Organización Mundial del Trabajo. (2012). Panorama OMT del turismo internacional. Edición 2012. Recuperado de http://www.e-unwto.org/doi/ pdf/10.18111/9789284414680

Organización Mundial del Comercio. (1995). Acuerdo General sobre el Comercio de Servicios (AGCS): objetivos, alcance $y$ disciplinas. Recuperado de https://www.wto.org/spanish/tratop_s/ serv_s/gatsqa_s.htm
Proexport. (2012). La Revista de las Oportunidades Proexport Colombia, Santander Aprovecha los TLC. Proexport: Bogotá.

Proexport. (2013). Informe de inversión extranjera directa en Colombia. Proexport: Bogotá.

República de Colombia. Ministerio de Comercio, Industria y Turismo - MinCIT. (2009). Desarrollando sectores de clase mundial en Colombia. Recuperado de http://www.mincit.gov.co/minindus tria/loader.php?lServicio=Documentos\&lFuncion=verPdf\&id=23172\&na me=2009-DesarrollandoSectoresEquipo.pdf\&prefijo=file

República de Colombia. DNP - Departamento Nacional de Planeación, (2007). Manual del empresario sobre las negociaciones comerciales de servicios de Colombia. Recuperado de http:// www.mincit.gov.co/loader.php?lServi cio=Documentos $\&$ lFuncion $=$ verPdf $\&$ id=19872\&name=ManualServicios. pdf\&prefijo=file 